\title{
Expression of P57 Immunohistochemical Marker in Complete And Partial Hydatidiform Mole by Using Tissue Microarray Technique
}

\author{
${ }^{1}$ Dr. Rehab K. Abbas. And ${ }^{2}$ Dr. Khitam R. Al-Khafaji \\ ${ }^{1,2}$ Department of pathology. College of Medicine. Baghdad University / Iraq
}

\begin{abstract}
:
Back ground: Hydatidiform mole is an abnormal form of pregnancy divided in to two types; complete hydatidiform mole and partial hydatidiform mole. Detailed histopathologic examination remains to be the basis for the diagnosis of hydatidiform mole (HM). However, poor sampling, necrosis, and earlier uterine evacuation can lead to uncertainty in the diagnosis. Also, the criteria are subjective, resulting in considerable interobserver variability. The P57KIP2 gene is paternally imprinted and maternally expressed, and the presence of its protein product serves as an adjuvant marker for the nuclear maternal genome. Because a complete HM is the only type of conceptus lacking a maternal contribution, P57 KIP2 immunostaining is correspondingly absent, whereas it is present in partial HM and normal pregnancy.

Aim of The Study: The purpose of this study is to evaluate the significance of the P57KIP2 immunohistochemical marker in the diagnosis of complete and partial hydatidiform mole, and to compare the P57KIP2 immunohistochemical marker results with that of ordinary Hematoxylin and Eosin slides histopathology results, in addition to evaluation of the Tissue Microarray Technique.

Materials and Methods: Seventy cases of endometrial biopsies were obtained. Histologic evaluation of all

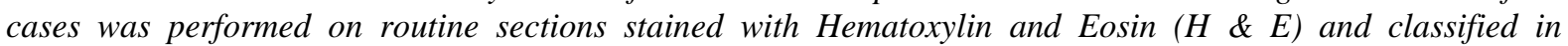
complete and partial hydatidiform mole (30 each) and (10 cases) of normal product of pregnancy (abortion), and negative control slides were also used. These cases were collected during the period from December-2011 to Augest-2012. New technique is adopted in this research that is; Tissue microarray in which twelve small cores of representative tissue samples, each measure $3 \mathrm{~mm}$ in diameter sections from microarray block are cut using a microtome, mounted on a single microscope slide and then analyzed by staining with Hematoxylin and Eosin, then another section made for the immunohistochemical staining with P57 KIP2 antibody.

Results: Regarding the expression of p57KIP2 IHC Marker in cases of PHM diagnosed previously by H\&E, 25 cases out of 30 cases which represented $83.3 \%$ expressed positive results. The remaining 5 cases which represented $16.7 \%$ expressed negative results, this means that they were definitely CHM. These findings were statistically highly significant.The results of P57KIP2 IHC Marker expression in cases of CHM diagnosed previously by $H \& E$, were 26 cases out of 30 cases which represented $86.7 \%$, expressed negative results this confirm the CHM diagnosis. The remaining 4 cases which represented $13.3 \%$, expressed positive results this means that they were most probably PHM. These findings were statistically highly significant.The age distribution of the patients in different grades of the CHM which expressed negative results for the IHC Marker p57KIP2 (Mean $=28.5$, median $=27$, mode $=25$ years old), it was found that the majority of patients in grade I were from age 20 to 39 years old, and the majority of patients in grade II were from age 20 to 29 years old, while the only one case of grade III was 38 years old. The correlation between grades of CHM and patients ages was not significant.The grades of the CHM which are negative for the IHC Marker p57KIP2, the majority of the patients were grade II which represent $53.4 \%$ and the minority of the patients were grade III which represent $3.3 \%$. It was noticed that all four cases which expressed positive immunostaining for the IHC marker p57KIP2 were; grade I, and they represented 13.3\%. The correlation between the grades of the CHM and the results of the p57KIP2 IHC marker was statistically significant.
\end{abstract}

Conclusion: P57KIP2 IHC marker is a useful adjunct, providing a definitive diagnosis of CHM.

Errors in morphologic analysis resulted primarily in over diagnosis of PHM are more frequent than in CHM.

There are significant differences in correct classification of hydatidiform mole between using P57KIP2 IHC marker and using $H \& E$ stain Cases in grade I of CHM were correlated significantly with the errors in morphological analysis resulted primarily in over diagnosis of CHM. Tissue Microarray is a relatively simple method can be used in pathology laboratories to decreased assay volume, time and cost.

Key words: Hydatidiform mole, P57KIP2, Tissue Microarray . 


\section{Introduction}

Hydatidiform mole (HM) is an abnormal pregnancy in which the placenta contains grape like vesicles (small sacs) that are usually visible with the naked eye. The vesicles arise by distention of the chorionic villi by fluid. When inspected in the microscope, hyperplasia of the trophoblastic tissue is noted. If left untreated, a hydatidiform mole will almost always end as a spontaneous abortion . Based on morphology, hydatidiform moles can be divided into two types: Complete hydatidiform moles (CHM), where all the chorionic villi are vesicular, and no sign of embryonic or fetal development is present. And partial hydatidiform moles (PHM) some villi are vesicular, whereas others appear more normal, and embryonic development may be seen but the fetus is always malformed and is never viable. Hydatidiform moles are common complication of pregnancy, occurring once in every 1000 pregnancies in the US, with much higher rates in Asia (e.g. up to one in 100 pregnancies in Indonesia). ${ }^{[1]}$ Immunohistochemical marker P57KIP2 is used recently in the diagnosis of HM, p57KIP2 is the protein product of the paternally imprinted but maternally expressed gene CDKN1C located on chromosome 11p15.5. Because CHM lack a maternal genomic component, they are not expected to express imprinted genes that are normally expressed by the maternal allele, and immunohistochemical analysis for p57 has been shown to be a valuable tool in the diagnosis of a CHM. However, immunohistochemical analysis for p57 cannot distinguish a PHM from hydropic abortion. ${ }^{[2]}$

\section{Materials and Methods}

Seventy cases of endometrial biopsies were obtained by curettage. Histologic evaluation of all cases was performed on routine sections stained with Hematoxylin and Eosin $(\mathrm{H} \& \mathrm{E})$ and classified in complete and partial hydatidiform mole (30 each) and (10 cases) of normal product of pregnancy (abortion), and negative control slides were also used by omission of primary antibody application from the staining procedure .

These cases were collected during the period from December/2011 to August/2012. The accompanying reports were reviewed for clinical information which included age of the patients and grades of complete hydatidiform mole. Mouse Monoclonal antibodies (MCAbs) used in this research was p57KIP2 (Kp10) produced by CELL MARQUE antibody company, California USA. New technique is adopted in this research that is; Tissue microarray which is done by set shown in ( fig 1). In the tissue microarray technique, a hollow needle is used to remove tissue cores as small as $3 \mathrm{~mm}$ in diameter from regions of interest, (regions of trophoblastic villi detected previously in the hematoxylin and eosin staining slides) in paraffin-embedded tissues (fig 2). These tissue cores are then inserted in a recipient paraffin block in a precisely spaced, array pattern. A microarray recipient paraffin block contains twelve small cores of representative tissue samples, each measure $3 \mathrm{~mm}$ in diameter (fig 3). Sections from microarray block are staining with hematoxylin and eosin, then an other section made for the immunohistochemical stain (fig 4). Statically analysed the results by Statistical Package for Social Sciences v15.0 (SPSS Inc., Chicago, IL, USA) software for Windows using Chi-square and Fisher's exact test. $P$ value $<0.05$ was considered significant.

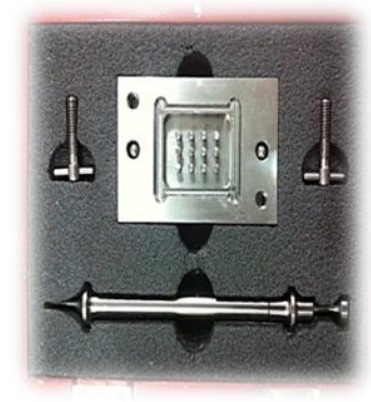

Fig 1)

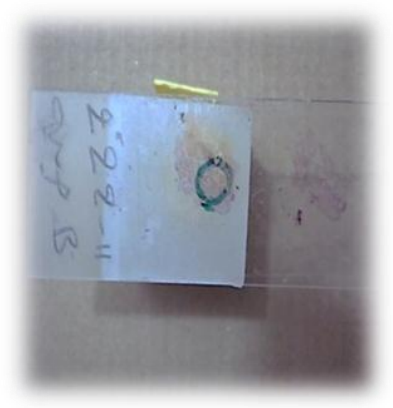

(fig 2)

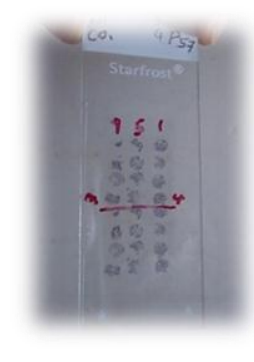

( fig 3)

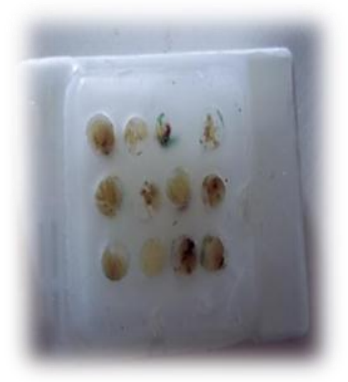

(fig 4)

\section{Results}

Considering the age distribution for all cases diagnosed by H\&E in this study, the actual age range was from 17 to 50 years old, and the age distribution were; (mean 29 years old; median 27 years old; mode 20 years old), the majority of the cases (50\%) were between the age of (20-29) years old and the minority of the cases $(10 \%)$ were younger than (19) years old . The correlation between the age of the patients and the type of the Hydatidiform Mole (HM) was not significant at the level 0.05 of $\mathrm{P}$ value, as shown in Table (1). 
Table (1): The correlation between the age of the patients and the type of the Hydatidiform Mole

\begin{tabular}{|c|c|c|c|c|c|c|c|c|c|c|}
\hline & \multicolumn{10}{|c|}{ Age of the patients } \\
\hline \multirow{2}{*}{$\begin{array}{c}\text { Hematoxylin } \\
\text { \&Eosin }\end{array}$} & \multicolumn{2}{|c|}{$10-19 \mathrm{yrs}$} & \multicolumn{2}{|c|}{$20-29$ yrs } & \multicolumn{2}{|c|}{$30-39 \mathrm{yrs}$} & \multicolumn{2}{|c|}{$40-50 \mathrm{yrs}$} & \multicolumn{2}{|c|}{ Total } \\
\cline { 2 - 13 } & No. & $\%$ & No. & $\%$ & No. & $\%$ & No. & $\%$ & No. & $\%$ \\
\hline Complete HM & 5 & 7.1 & 13 & 18.6 & 8 & 11.4 & 4 & 5.7 & 30 & 42.9 \\
\hline Partial HM & 0 & 0 & 17 & 24.3 & 7 & 10.0 & 6 & 8.6 & 30 & 42.9 \\
\hline Abortion & 2 & 2.9 & 5 & 7.1 & 3 & 4.3 & 0 & 0 & 10 & 14.2 \\
\hline Total & 7 & 10.0 & 35 & 50.0 & 18 & 25.7 & 10 & 14.2 & 70 & 100 \\
\hline
\end{tabular}

Pearson chi-square $[\mathrm{df}=6]: 8.148$

$P$ value: 0.227

Statistical test: Not significant

Regarding the grades of the CHM based on the H\&E category the majority of the patients were grade II which represents $53.3 \%$ and the minority of the patients were grade III which represents $3.3 \%$ as shown in Table (2).

Table (2): The nomber of cases with different grades of the complete mole

\begin{tabular}{|c|c|c|c|c|c|c|c|c|}
\hline GRADE of Complete HM & \multicolumn{2}{|c|}{ I } & \multicolumn{2}{|c|}{ II } & \multicolumn{2}{|c|}{ III } & \multicolumn{2}{c|}{ TOTAL } \\
\hline $\begin{array}{c}\text { Complete HM Morphology } \\
\text { In Hematoxylin \&Eosin } \\
\text { Staining }\end{array}$ & No. & $\%$ & No. & $\%$ & No. & $\%$ & No. & $\%$ \\
\cline { 2 - 10 } & 13 & 43.3 & 16 & 53.4 & 1 & 3.3 & 30 & 100 \\
\hline
\end{tabular}

The correlation between the age of the patients and the grades of the CHM based on H\&E morphology The age distribution of the patients in the different grades of the CHM based on H\&E category were ;

( Mean $=28$, median=27, mode $=25$ years old). The majority of the patients in grade I and grade II were from 20 to 29 years old (20\% each) and the minority were from $40-50$ years old $(3.3 \%, 10 \%)$ respectively . The correlation between the age of the patients and the grades of the CHM was not significant at the 0.05 level of $\mathrm{P}$ value, as shown in Table (3).

Table (3): The correlation between the age of the patients and the grades of the CHM

\begin{tabular}{|c|c|c|c|c|c|c|c|c|c|c|}
\hline \multirow{3}{*}{$\begin{array}{l}\text { GRADE of Complete } \\
\text { HM } \\
\text { Morphology } \\
\text { By H\&E }\end{array}$} & \multicolumn{10}{|c|}{ Age of patients } \\
\hline & \multicolumn{2}{|c|}{$10-19 \mathrm{yrs}$} & \multicolumn{2}{|c|}{$20-29$ yrs } & \multicolumn{2}{|c|}{$30-39$ yrs } & \multicolumn{2}{|c|}{$40-50$ yrs } & \multicolumn{2}{|c|}{ TOTAL } \\
\hline & No. & $\%$ & No. & $\%$ & No. & $\%$ & No. & $\%$ & No. & $\%$ \\
\hline Grade I & 2 & 6.7 & 6 & 20.0 & 4 & 13.3 & 1 & 3.3 & 13 & 43.3 \\
\hline Grade II & 3 & 10.0 & 6 & 20.0 & 4 & 13.3 & 3 & 10.0 & 16 & 53.4 \\
\hline Grade III & 0 & 0 & 0 & 0 & 1 & 3.3 & 0 & 0 & 1 & 3.3 \\
\hline TOTAL & 5 & 16.7 & 12 & 40.0 & 9 & 30.0 & 4 & 13.3 & 30 & 100 \\
\hline
\end{tabular}

Pearson chi-square $[\mathrm{df}=6]: 3.334$

$P$ value: 0.766

Statistical test: Not significant

Regarding the IHC P57KIP2 Marker immunostaining in the normal product of pregnancy, its found that all cases express positive results $(100 \%)$ as shown in Table (4).

Table (4): The expression of P57KIP2 IHC marker in abortion cases

\begin{tabular}{|l|l|l|}
\hline & \multicolumn{2}{|l|}{ Cases of Abortion } \\
\hline H\&E Ordinary stain & 10 & $100 \%$ \\
\hline P57 IHC Marker +ve result & 10 & $100 \%$ \\
\hline P57 IHC Marker -ve result & 0 & $0 \%$ \\
\hline
\end{tabular}

The immunostaining of p57KIP2 IHC marker in the PHM cases diagnosed previously by H\&E, were; 25 cases out of 30 cases which represented $83.3 \%$ expressed positive results and 5 cases out of 30 cases which represented $16.7 \%$ expressed negative results, this means that they were definitely CHM. These findings were statistically highly significant at the level 0.05 of $\mathrm{P}$ value, as shown in Table (5). 
Expression of P57 Immunohistochemical Marker In Complete And Partial Hydatidiform Mole ...

Table (5): The expression of P57KIP2 IHC Marker in cases of PHM diagnosed previously by H\&E

\begin{tabular}{|l|c|c|c|c|c|c|}
\hline \multirow{2}{*}{} & \multicolumn{2}{|c|}{ Partial HM } & \multicolumn{2}{c|}{ Complete HM } & \multicolumn{2}{c|}{ TOTAL } \\
\cline { 2 - 7 } & No. & $\%$ & No. & $\%$ & No. & $\%$ \\
\hline H\&E Ordinary stain & 30 & 100 & 0 & 0 & 30 & 100 \\
\hline P57 IHC Marker +ve result & 25 & 83.3 & 0 & 0 & 25 & 83.3 \\
\hline P57 IHC Marker -ve result & 0 & 0 & 5 & 16.7 & 5 & 16.7 \\
\hline
\end{tabular}

Pearson chi-square $[\mathrm{df}=6]: 60.000$

P value: 0.000

Statistical test: Highly significant.

The immunostaining results of p57KIP2, IHC marker in the CHM cases diagnosed previously by H\&E, were; 26 cases out of 30 cases which represented $86.7 \%$, were expressed negative results this confirm the CHM diagnosis. The remaining 4 cases which represented $13.3 \%$, were expressed positive results, this means that they are most probably PHM. These findings were statistically highly significant at the level 0.05 of $\mathrm{P}$ value.

The age distribution of the patients in the different grades of the CHM which expressed negative results for the IHC Marker P57KIP2 were; ( Mean=28.5, median=27, mode=25 years old). Its found that the majority of patients in grade I were from age 20 to 39 years old, and the majority of patients in grade II were from age 20 to 29 years old, while the only one case of grade III was 38 years old, the correlation between grades of CHM and age of patients was not significant at the level 0.05 of $\mathrm{P}$ value,

In our study the cases of the CHM which expressed negative results for the IHC Marker P57KIP2 were 26 cases out of 30 cases, the majority of the patients were grade II which represented $53.4 \%$ and the minority of the patients were grade III which represented 3.3\%. It was noticed that all four cases which expressed positive immunostaining for the IHC Marker P57KIP2 were grade I, and they represented 13.3\%. The correlation between the grades of the CHM and the results of the IHC P57KIP2 marker was statistically significant at the level 0.05 of $\mathrm{P}$ value, as shown in Table (6).

Table (6): The correlation between the Grades of the CHM and the results of the IHC marker P57KIP2.

\begin{tabular}{|c|c|c|c|c|c|c|c|c|}
\hline \multirow[t]{2}{*}{ GRADE of Complete HM } & \multicolumn{2}{|c|}{$\mathrm{I}$} & \multicolumn{2}{|c|}{ II } & \multicolumn{2}{|c|}{ III } & \multicolumn{2}{|c|}{ TOTAL } \\
\hline & No. & $\%$ & No. & $\%$ & No. & $\%$ & No. & $\%$ \\
\hline P57 Immunohistochemical marker -ve result & 9 & 30.0 & 16 & 53.4 & 1 & 3.3 & 26 & 86.7 \\
\hline
\end{tabular}

Pearson chi-square $[\mathrm{df}=6]: 60.036$

P value: 0.049

Statistical test: Significant.

In this study the cases were stained with [H\&E] to demonstrate and re-evaluate features of normal and molar pregnancy morphologically, and compared with the observation of (IHC) marker P57KIP2 expression in the nucleus of cytotrophoblasts and stromal villus cells in these three different categories as illustrated bellow : 1-Normal product of pregnancy [Abortion] shown in figures:

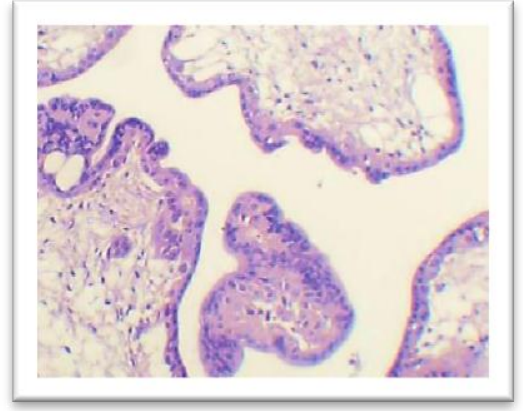

Fig (5) Abortion in Hematoxylin and eosin staining $\times 100$

$\times 100$

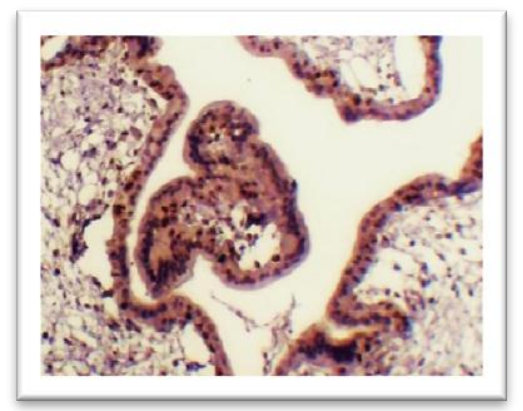

Fig (6) Abortion with immunohisto chemical positive staining for P57

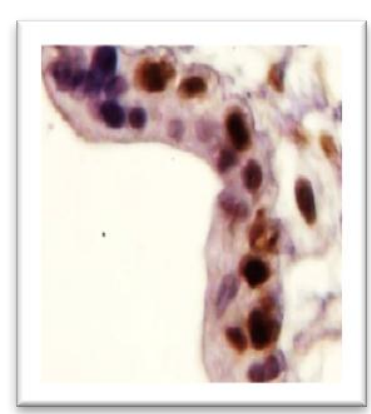

Fig (7) Positive staining nuclei for P57 in the strom $\times 400$ 
2-Parial HM shown in figures:

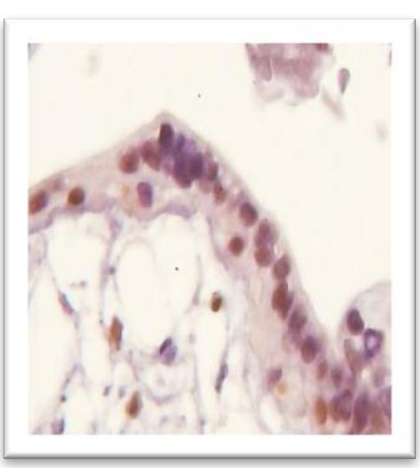

Fig (8) PHM in Hematoxylin and eosin staining $\times 100$ $\times 100$

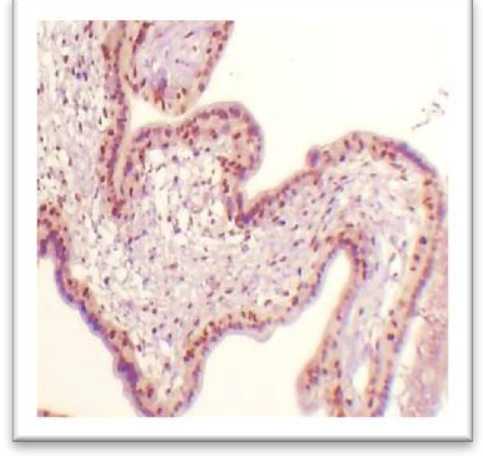

Fig (9) PHM with immunohisto chemical positive staining for P57 al cell in $\mathrm{PHM} \times 400$

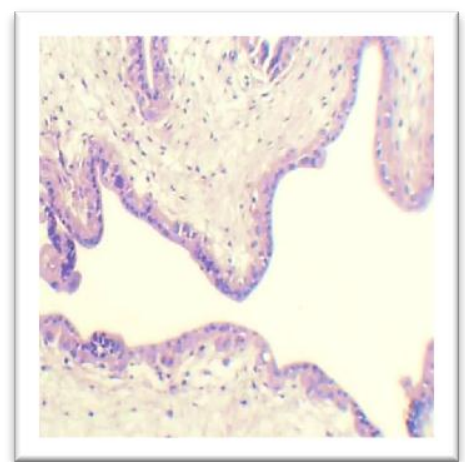

Fig (10) Positive staining nuclei for P57 in the strom 400

3-Complete HM shown in figures:

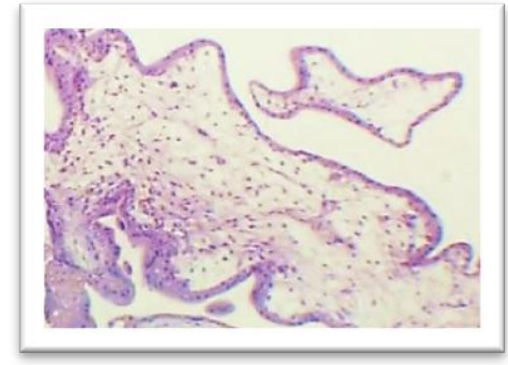

Fig (11) CHM in Hematoxylin and eosin staining $\times 100$ $\times 100$

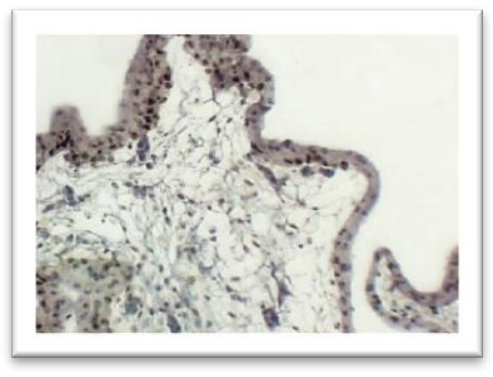

Fig (12) CHM with immunohisto chemical negative staining for P57

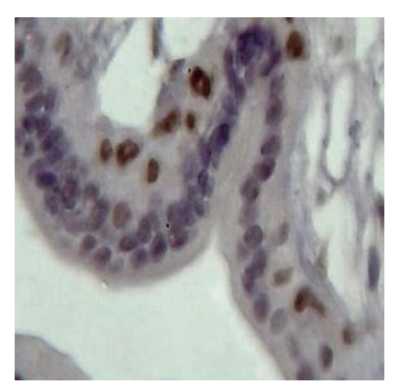

Fig (13) negative staining nuclei for P57 in the strom al cell in $\mathrm{CHM} \times 400$

\section{Discussion}

In our study of the 70 cases diagnosed as molar and normal pregnancies by H\&E, the actual age range was from 17 to 50 years old, and the age distribution were; (mean 29, median 27 , mode 20 years old), the majority of the cases $(50 \%)$ were between the age of (20-29) years old whereas only (10\%) of the cases were younger than (19) years old, the correlation between the age of the patients and the type of the Hydatidiform Mole (HM) was not significant. In a similar study, Shakil H. Merchant.et al. (2005), in USA, found that; Patients age ranged from 14 to 41 years old, the mean age was 24.3 years old). And there was no correlation between age of the patients and the disease. ${ }^{[3]}$

In a study of Pathomorphology of molar gestation in Zaria (2010), ${ }^{[4]}$ Mayun A. A. et al. said; "the age range for all the cases of molar pregnancies was 15 - 49 years old, with a mean of 25.7 years old". Mayun A A et al. found that the peak incidence was in the third decades of life which falls within the same range seen in parts of Nigeria ${ }^{[5]}$ and California. ${ }^{[191]}$ This however is lower than reports from the Far East, where patients over 40 years of age were most prone to develop the disease. ${ }^{[6]}$

Surely the age of the patients in similar studies is during the reproductive age of the females therefore, its rarely to find (HM ) below 13 and above 55 years old.

Grades of patients with CHM based on H\&E category were; $43.3 \%$ grade I, $53.4 \%$ grade II, and $3.3 \%$ grade III, and the age distribution for each grade was not statistically correlated.

In an Iraqi study regarding Placental Trophoblastic Diseases, 30 cases of CHM were taken, from which $33.3 \%$ were grade I, $33.3 \%$ were grade II, and $33.3 \%$ were grade III. However, their age distribution was also not statistically correlated with the grades. ${ }^{[7]}$

Murphy KM. et al. (2009) in USA, claimed that; "Nuclear p57KIP2 expression is seen in villous cytotrophoblasts and stromal cells in normal placenta. Moreover, PHM with maternal genome shows p57KIP2 expression, Which in contrast is completely absent in villous cytotrophoblasts and stromal cells of CHM as they do not possess maternal genomic DNA".$^{[8]}$ In the present study normal placentas (Abortion) showed strong and contiguous p57KIP2 nuclear expression in cytotrophoblast and villous mesenchyme, However Dorota A. Popiolek et al. (2006) in New York found that 10 out 10 cases of normal placentas strongly expressed p57KIP2 in the cytotrophoblasts and in villus stromal cells. ${ }^{[9]}$ 
M. Paul et al.(2010) in $\mathrm{USA}^{[10]}$ found that all cases of normal product of conception which were 10 cases shows strong positivity for p57KIP2 IHC marker.

Regarding the expression of p57KIP2, IHC Marker in cases of PHM diagnosed previously by H\&E, 25 cases out of 30 cases which represented $83.3 \%$ expressed positive results. The remaining 5 cases which represented $16.7 \%$ expressed negative results, this means that they were definitely CHM. These findings were statistically highly significant. The results of p57KIP2 IHC Marker expression in cases of CHM diagnosed previously by H\&E, were 26 cases out of 30 cases which represented $86.7 \%$, expressed negative results this confirm the CHM diagnosis. The remaining 4 cases which represented $13.3 \%$, expressed positive results this means that they were most probably PHM. These findings were statistically highly significant. In similar study, $\mathrm{H}$ Crisp et al.(2003) in UK said; The concordance between histological diagnosis and p57KIP2 was very good All 16 partial moles were p57KIP2 immunoreactive, and twenty of the $22(90.9 \%)$ complete moles showed no immunoreactivity for $\mathrm{p} 57 \mathrm{KIP} 2$. The remaining two cases showed nuclear immunoreactivity in villous cytotrophoblast. ${ }^{[1]}$ Shao-min Yang et al.(2009) in China, found in 32 cases of (HM) that 21 out of 22 case of CHM were p57KIP2 negative, whereas all the 11 cases of PHM were positive (11/11). ${ }^{[12]}$

Hanène Landolsi et al. in a study in Tunisia (2011) agreed with the previously mentioned results in which p57KIP2 protein was investigated in 220 specimens of first trimester hydropic abortuses (HA), and it was compared with the original diagnosis based on morphology, including 132 CHM, 49 PHM, and 39 HA. Concordant results were obtained in 210 cases. In 9 of 10 cases with a discordant diagnosis; negative immunostaining in 8 cases morphologically diagnosed as PHM and one case diagnosed as (HA), the remaining case was morphologically diagnosed as CHM and expressed positive immunostaining for p57KIP2. ${ }^{[13]}$ In Iran S. Sarmadi et al.(2011) 54 cases of HM, found that 22 out of 22 cases of morphologically diagnosed CHM were expressed negative P57KIP2 results and 25 out of 32 cases (78.1\%) of morphologically diagnosed PHM were expressed positive P57KIP2 results and only 7 cases (21.9\%) were showed negative immunostaining for P57KIP2. ${ }^{[14]}$

Conclusion: P57KIP2 IHC marker is a useful adjunct, providing a definitive diagnosis of CHM.

Errors in morphologic analysis resulted primarily in over diagnosis of PHM are more frequent than in CHM.

There are significant differences in correct classification of hydatidiform mole between using P57KIP2 IHC marker and using $H \& E$ stain Cases in grade I of CHM were correlated significantly with the errors in morphological analysis resulted primarily in over diagnosis of CHM. Tissue Microarray is a relatively simple method can be used in pathology laboratories to decreased assay volume, time and cost.

References

[1]. Di Cintio E, Parazzini F, Rosa C, et al. "The epidemiology of gestational trophoblastic disease". Gen Diagn Pathol 143 (2-3) (1997): 103-8.

[2]. Robin D. LeGallo, MD,1 Edward B. Stelow, MD,1 et al. " Diagnosis of Hydatidiform Moles Using p57 Immunohistochemistry and HER2 Fluorescent In Situ Hybridization". Am J Clin Pathol;129(2008):749-755 .

[3]. Shakil H. Merchant, Mitual B. Amin, David S. Viswanatha, p57KIP2 immunohistochemistry in early molar pregnancies: emphasis on its complementary role in the differential diagnosis of hydropic abortuses Human Pathology (2005) 36, 180- 186.IVSL.

[4]. AA Mayun, AH Rafindadi, MS Shehu,et al. "Pathomorphology of molar gestation in Zaria", NMJ $2010: 51,1,: 1-4$.

[5]. Ekpo M. D. Pathomorphology and clinical correlation of hydatidiform moles. FMCPath thesis. National Postgraduate Medical College of Nigeria. May 1988.

[6]. Buckley J. The epidemiology of molar pregnancy and choriocarcinoma. Clin Obstet Gynaecol 1984; 27(1): 153-159.

[7]. Zainab M. AL.shamma'a, Placental trophoblastic diseases immunohistochemical stains and (Ag-NORs) study, 2001.P:33-42.

[8]. Murphy KM, Hafez M, McConnell TG, et al, Ronnett BMD Diagnosis and subclassification of hydatidiform molesusing p57 immunohistochemistry and molecular genotyping: validation and prospective analysis in routine and consultation practice setting with development of an algorithmic approach. Am J Surg Pathol (2009) 33:805-817. IVSL.Dorota A. Popiolek, Herman Yee, Khush Mittal, et al. Multiplex short tandem repeat DNA analysis confirms the accuracy of p57KIP2

[9]. immunostaining in the diagnosis of complete hydatidiform mole. Human Pathology (2006) 37, 1426- 1434.

[10]. Maureen Paula , Suzan Goodmanc, Juan Felixd et al. Early molar pregnancy: experience in a large abortion service. 81 (2010)150156.IVSL.

[11]. Crisp H., Burton J. L., Stewart R. and Wells, M. ,Refining the diagnosis of hydatidiform mole: image ploidy analysis and p57KIP2 immunohistochemistry. Histopathology, October 2003 43: 363-373.

[12]. Shao-min Yang, Jiang-feng You, Xiao-wei Zhang, et al. Roles of p57KIP2 immunohistochemistry and flow cytometry in diagnosis of molar pregnancy 2009 Sep;38(9):580-4.

[13]. Hanène Landolsi, Nabiha Missaoui, Sonia Brahem, et al. The usefulness of p57(KIP2) immunohistochemical staining and genotyping test in the diagnosis of the hydatidiform mole. Pathol Res Pract.J 2011 Aug 15;207(8):498-504

[14]. Soheila Sarmadi, Narges Izadi-Mood, Ali Abbasi et al p57KIP2 immunohistochemical expression: a useful diagnostic tool in discrimination between complete hydatidiform mole and its mimics, Arch Gynecol Obstet. 2011 Apr;283(4):743-8.IVSL. 